\title{
A review of probabilistic analysis in orthopaedic biomechanics
}

\author{
P J Laz ${ }^{1 *}$ and M Browne ${ }^{2}$ \\ ${ }^{1}$ Computational Biomechanics Lab, Department of Mechanical and Materials Engineering, University of Denver, \\ Denver, Colorado, USA \\ ${ }^{2}$ Bioengineering Sciences Research Group, School of Engineering Science, University of Southampton, Highfield, \\ Southampton, UK
}

The manuscript was received on 18 September 2009 and was accepted after revision for publication on 15 December 2009.

DOI: 10.1243/09544119JEIM739

\begin{abstract}
Probabilistic analysis methods are being increasingly applied in the orthopaedics and biomechanics literature to account for uncertainty and variability in subject geometries, properties of various structures, kinematics and joint loading, as well as uncertainty in implant alignment. As a complement to experiments, finite element modelling, and statistical analysis, probabilistic analysis provides a method of characterizing the potential impact of variability in parameters on performance. This paper presents an overview of probabilistic analysis and a review of biomechanics literature utilizing probabilistic methods in structural reliability, kinematics, joint mechanics, musculoskeletal modelling, and patient-specific representations. The aim of this review paper is to demonstrate the wide range of applications of probabilistic methods and to aid researchers and clinicians in better understanding probabilistic analyses.
\end{abstract}

Keywords: probabilistic methods, biomechanics, joint mechanics, kinematics, total joint replacement, statistics, finite element analysis

\section{INTRODUCTION}

Uncertainty and variability are present in many aspects of biomechanics and orthopaedics; factors such as patient geometry, material properties of various structures, kinematics and joint loading, implant design and component alignment, as well as clinical outcomes are all variable in nature. As a result, the use of statistical analysis has long been the standard in the field. Anthropometric measures have been characterized with distributions and correlations; Student's $t$ tests and analysis of variance (ANOVA) have been performed to assess statistically significant differences in experimental data. Sensitivity of a system has been assessed with parametric studies, perturbing individual parameters, and design of experiments (DOE), utilizing a matrix of tests or analyses with parameters set at

*Corresponding author: Computational Biomechanics Lab, Department of Mechanical and Materials Engineering, University of Denver, 2390 South York Street, Denver, CO 80208, USA. email: plaz@du.edu various levels to account for interaction effects (Fig. 1). Sensitivity studies determine which of the parameters considered has the most influence on the outcome of the system when it's varied. Such studies have been applied widely, particularly in computational studies; however, these methods often exclude statistical information about the probability distribution for the input factors by taking trials evenly sampled across an input range. Similarly, parametric sweep studies (where a particular factor is varied across a range of values) fail to map out the entire design space, and while such studies are an essential first step, they provide no information about the effect of variability.

In contrast to a deterministic study where an output can be exactly determined from a set of inputs, probabilistic analysis attempts to predict the output (and its likelihood) accounting for uncertainty associated with the inputs. Consider, for example, the response of an implanted hip. A deterministic analysis is likely to assess the potential differences in the response for a large, active patient and a small, elderly patient. However, there are often 

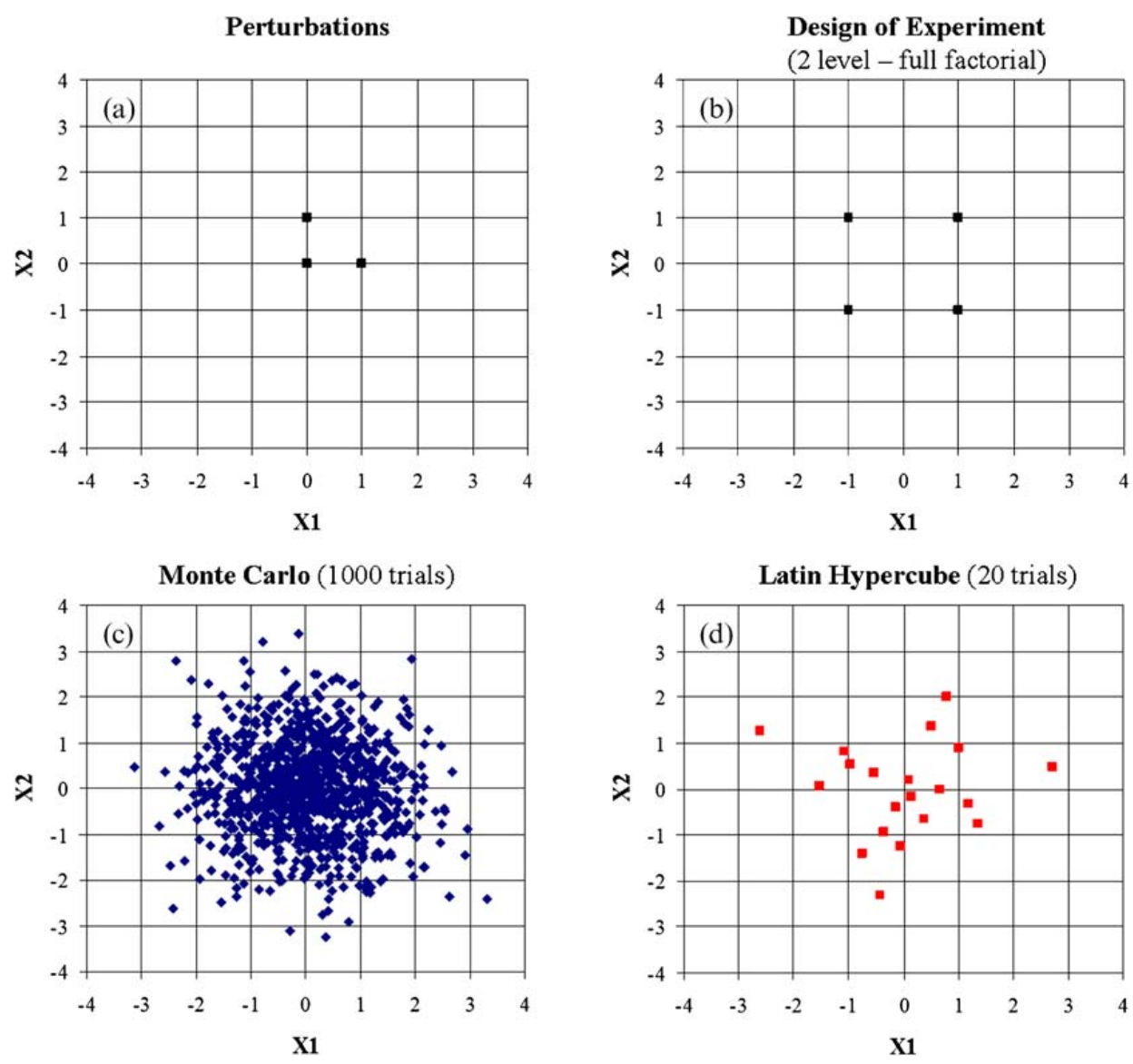

Fig. 1 Representation of sample points for two variables with (a) perturbation-based sensitivity, (b) design of experiment with two levels, (c) Monte Carlo analysis with 1000 trials, (d) Latin hypercube sampling with 20 trials

many other factors that could impact the hip's response in a specific patient, including bone quality, location and alignment of the implant, integrity of the surrounding soft tissue structures, and musculature. Many of these factors are difficult to quantify and may not be known explicitly, yet have the potential to impact the response of the hip. If enough information is known about the system and its input parameters, it is possible to use statistical methods to quantify the response of the system. As the system becomes more complex, increasingly sophisticated methods are required to account for the various sources of uncertainty, as is often the case in orthopaedic biomechanics.

In probabilistic studies, each of the input parameters is represented as a distribution instead of a single mean value. A distribution can be defined by a probability density function (PDF) or histogram indicating the likelihood of the parameter taking on a specific value. Alternatively, a distribution can be represented by a cumulative distribution function (CDF), which involves ordering the data from the smallest to the largest and evenly spacing them between 0 and 1 on the ordinate [1]. A distribution may take any form, but a number of common types are encountered (e.g. normal or Gaussian, lognormal, Poisson, binomial, Weibull). Once defined, probabilistic methods use the input distributions to predict a distribution of performance; the distribution characterizes the range of possible outcomes and their likelihood, which leads to an understanding of the probable outcomes. The resulting distribution can be used to assess the bounds of performance, including performance associated with a specific level of risk or risk associated with a specific level of performance. In addition, sensitivity factors, commonly computed as part of the probabilistic analysis, can provide insight into which parameters are affecting performance and to what extent. By representing uncertainty in multiple inputs, potential interaction effects are incorporated and the evaluation is more robust than sensitivity studies performed by varying parameters individually. 
Probabilistic methods have been extensively applied in biomechanics applications. Monte Carlo simulation, which involves randomly generating values for each variable according to its distribution and then predicting the distribution of performance through repeated trials, remains the most commonly applied probabilistic method (Fig. 1). While robust, Monte Carlo simulation is computationally expensive and a variety of other more efficient probabilistic methods have been developed. Generally, these methods improve efficiency by either reducing the sample space based on knowledge of the system or they are more approximate. While details of these methods can be found in textbooks $[\mathbf{1}, 2]$ and statistics journals, this paper seeks to present probabilistic modelling in the context of the unique applications in the biomechanics field.

Accordingly, the objective of this review paper is to present the probabilistic methods and analysis techniques as they are commonly used in biomechanics applications, demonstrating the wide range of applications and aiding the scientific community in understanding these methods, their benefits, as well as limitations. The paper is organized by presenting a brief overview of methods and commonly used tools, followed by common applications in structural reliability, kinematics, joint mechanics, musculoskeletal modelling, and patient-specific representation.

\section{PROBABILISTIC ANALYSIS METHODS}

\subsection{Overview of analysis methods}

The most commonly applied probabilistic method is Monte Carlo simulation, which involves repeated randomly sampling of variables according to their distributions to populate a distribution of performance. Typically many thousands of trials are required to obtain useful results, and the number of trials required will increase when the probabilities involved are small. While Monte Carlo simulation is often referred to as the 'gold standard', it should be noted that the accuracy of the results are dependent on the number of trials; associated sampling errors are dependent on the probability level and can be computed [1]. A strength of the Monte Carlo method is its ability to converge to the correct solution, even if it may be computationally expensive.

A variation of Monte Carlo simulation that involves more efficiently controlling the sampling is Latin hypercube sampling (LHS). Rather than distribute the trials entirely at random across the possible sample space, LHS attempts to ensure an even coverage of the design space by partitioning it such that the associated probability of each partition is equal [1]. Thus larger partitions or samples exist at the tails of the distributions and more partitions at the peaks, thus reducing the risk of clustering of samples (Fig. 1). For problems with multiple dimensions, samples are selected to give a good statistical spread by ensuring that each sample falls into a unique row and column. The two-dimensional case is called 'Latin square' sampling, while an $N$ dimensional case is known as 'Latin hypercube' sampling. It should be noted that for problems with multiple dimensions, the LHS method can still result in clustering. In addition, as the number of trials increases, the possibility of clustering decreases with Monte Carlo and the benefits of LHS are reduced.

Another adaptation of Monte Carlo simulation is the importance sampling method (ISM). With ISM, the design space is not fully explored and instead sampling focuses on areas of interest. The effect is to multiply the accuracy; for example, if it is known that three-quarters of the design space will not be associated with a certain performance outcome then samples can be focused in the remaining quarter, such that the same accuracy is achieved 4 times faster.

Response surface methods (RSMs) fit a simple analytical function of the input variables to approximate the output parameter over the full range of the sample space. Typically, this will be a low-order polynomial equation (the response surface equation, RSE) and regression techniques will be used to select the term coefficients. Once an RSE is derived, this can be used as the basis for a Monte Carlo simulation, since it can be evaluated much faster than the true model. This method works best when the true output is well represented by the analytic function. This is the case for very linear models; however, highly non-linear functions may not be well represented and the higher the order of the RSE, the more samples are needed to achieve a good fit with the regression. A limitation of this technique is that results do not generally achieve a high degree of accuracy because the same analytic function must approximate the output across the entire sample space.

In order to reduce analysis time, especially when deterministic model run times are long, a series of approximate most probable point (MPP) methods have been utilized that are more computationally efficient $[\mathbf{1}, \mathbf{3}]$. The MPP represents the combination of input parameter values that predict performance 
at a specified probability level (Fig. 2), where performance often represents failure in structural reliability applications. The MPP methods typically determine the most probable point using optimization on a first-order Taylor series approximation of the performance function [1]. Some implementations map the original random variables into independent standard normal variables to facilitate optimization with variables of similar magnitudes. The various techniques differ in terms of how the MPP is computed; for example, the FORM (firstorder reliability method) and SORM (second-order reliability method) use a first-order or second-order approximation respectively. While the MPP methods are approximate, they have been shown in many analyses to be quite accurate in comparisons with Monte Carlo simulation results, while requiring a small fraction of the number of computations. The low computational cost of the MPP methods comes with a tradeoff; this method only provides information for a single point (e.g. probability), so in order to construct a full PDF or CDF, the method must be applied repeatedly at each point of interest. The MPP approaches can have difficulty dealing with highly non-linear limit state functions. It is important to highlight that the MPP methods require a well-behaved monotonic system; when multiple combinations of parameters result in the same output, the method has difficulty converging to a meaningful solution.

Further detailing the MPP family of methods, the mean-value (MV) method constructs a mean-based response function and computes the MPP for the specified probability levels. As a first-order method, it provides a good approximation of the solution near the mean. It is suitable for fairly linear problems, but can deviate significantly towards the tails for non-linear problems. The MV method requires $n+1$ trials, where $n$ is the number of random variables. The advanced mean-value (AMV) method utilizes the MV as a basis to achieve a better representation of the response. It does this by including corrective terms to approximate higherorder effects and requires $n+1+m$ trials, where $m$ is the number of specified probability levels [4]. Unlike the RSM, FORM, and SORM, the AMV method does not provide a parametric function that can be applied anywhere in the possibility space; instead, it takes the MV prediction and, using data from the calculated MPP of interest, corrects this value for a single level of desired probability (or desired output). The higher-order approximation achieved by AMV cannot be applied at any point other than that for which it was derived; getting estimates for additional points requires additional applications of the AMV method. The advanced mean-value with iterations
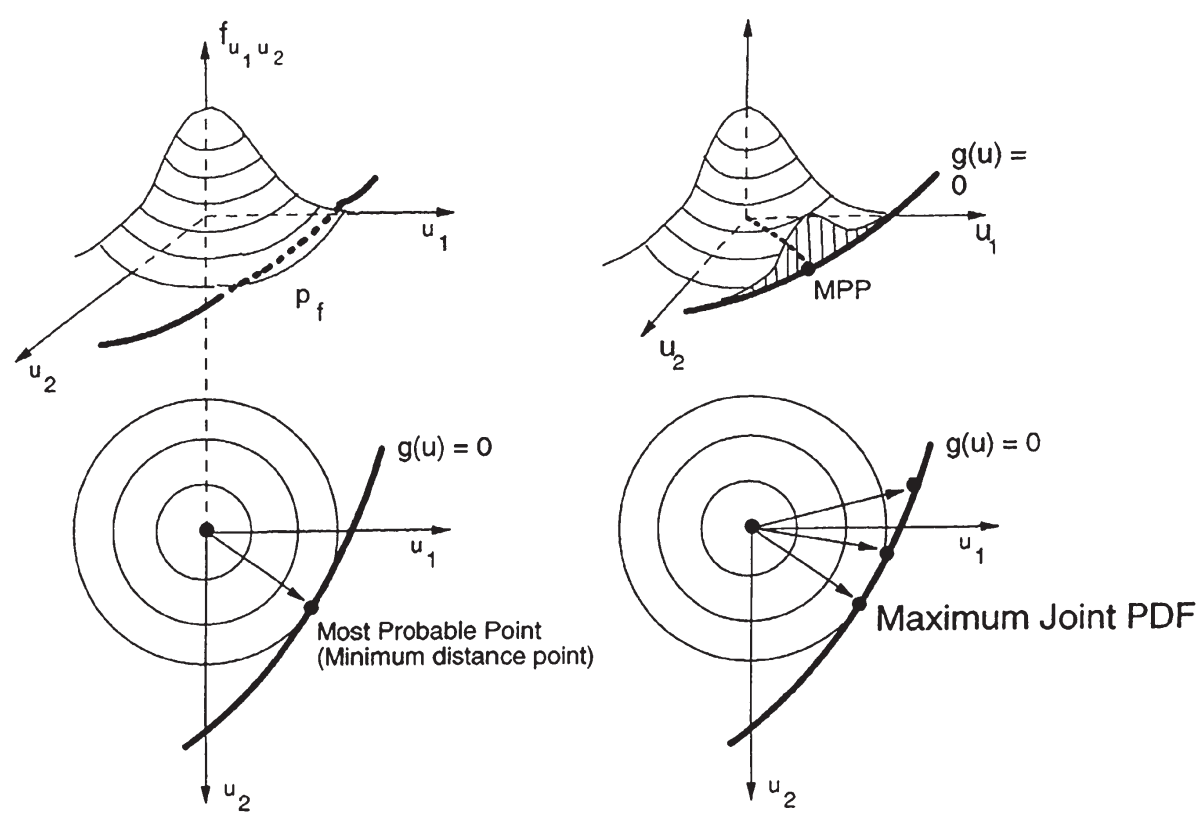

Fig. 2 The most probable point (MPP) methods use optimization to find the MPP along the limit state equation. The MPP represents the shortest distance to the origin in the standard normal space and the highest frequency along the limit state equation. (The figure is reprinted from NESSUS Theoretical Manual, 2001, with permission from Southwest Research Institute, San Antonio, Texas) 
(AMV+) method involves the implementation of AMV but also includes iterations on the MPP to ensure that convergence to a specified level is reached. AMV+ has been shown to be very accurate, even for non-linear problems, though the number of trials varies with the problem [4].

\subsection{Design sensitivity factors}

Design sensitivity factors are another valuable result of probabilistic analyses, as they indicate the effect of each individual parameter on a given system output. Knowledge of the most important parameters impacting performance is relevant to engineers, designers, and clinicians. Sensitivity factors can also serve as a useful guide on which factors to keep or remove in future probabilistic studies if computational resources are limited. In the case of predictions for a motion cycle (e.g. gait), sensitivity factors for each performance measure are often averaged over the cycle to provide a more straightforward ranking of the variables.

There are relative and absolute sensitivities, each with unique advantages. Relative sensitivities are commonly referred to as probabilistic importance factors, $\alpha$, and give the change in reliability index, $\beta$, with respect to the standard normal variate, $u$. The importance factor is defined as

$$
\alpha_{i}=\frac{\partial \beta}{\partial u_{i}}=\frac{\partial \beta}{\partial p} \frac{\partial p}{\partial u_{i}}
$$

for each variable with $p$ equal to a specific probability level. The reliability index, $\beta$, represents performance in the standard normal variate space, where, for example, probabilities of 0.001 to 0.999 are represented by standard normal variates of -3 to +3 . The sensitivity factor $\alpha$ is computed from the unit vector to the MPP and represents the relative contribution of each variable to the variability in performance. A positive sensitivity indicates a direct relationship between the value of the variable and the response, while a negative sensitivity indicates an inverse relationship. As the standard normal variate is a function of the mean, standard deviation, and distribution type, the $\alpha$ sensitivity factor is not always ideal for the design process.

Instead, absolute sensitivities, $S_{\mu}$ and $S_{\sigma}$, may be evaluated. These give the change in probability with respect to the mean and standard deviation respectively and are determined by

$$
S_{\mu}=\frac{\partial p}{\partial \mu_{i}} \frac{\sigma_{i}}{p}, S_{\sigma}=\frac{\partial p}{\partial \sigma_{i}} \frac{\sigma_{i}}{p}
$$

where the sensitivities are non-dimensional, allowing comparisons to be made between all of the variables [5]. These sensitivities indicate how much the mean and standard deviation of each random variable contribute to the variability in the response.

In Monte Carlo analyses, relationships between each variable and the performance measure can be used as a measure of sensitivity. Specifically, correlation coefficients serve as a measure of the strength of the relationship, while slopes assess how a change in input parameter affects the output. Correlation values near 1 or -1 indicate direct or inverse relationships (high sensitivity) between the input parameter and the output, while correlation values near 0 indicate no relationship (low sensitivity). Notably, a high correlation speaks to the level of agreement and can identify the parameters influencing performance, but slope is required to characterize how a change in an input variable affects performance. As they are linear measures, correlation coefficients and slopes may not provide a good representation of sensitivity in highly non-linear systems.

\subsection{Common challenges}

The most common challenges in probabilistic analysis are the number of analyses or evaluations required and determining the appropriate input parameter representations. Whether performing a patient-specific analysis or an implant design phase evaluation, achieving the solution in a timely manner is critical. When performing a probabilistic analysis, the repeated model evaluations will require more computation time than a deterministic evaluation, but are justified in many cases by the additional information gained. In general, there are typically tradeoffs between accuracy and efficiency; the efficient sampling and approximate techniques described in the previous section can provide accurate and timely solutions, but may require validation for a specific system.

Input parameters are represented by a distribution type (e.g. normal, lognormal, Weibull) and the associated parameters, which should be derived from measured data when available. The appropriate selection of the input parameters is especially important as it directly affects the predicted bounds of performance and sensitivity factors. For material properties, for example, the distribution type and parameter values (mean, standard deviation) can be determined from an experimental dataset. However, input data for some parameters, like component 
alignment, friction, or dimensional tolerances, are often not known, not available, and/or difficult to measure. In these cases, distributions can be estimated based on bounds and tolerances.

In contrast to material properties or applied loading, which can be easily varied in the model when the distribution is known, implementing geometric changes is usually more challenging as such changes often require remeshing the model geometry. In structural reliability analyses, for example, it is desirable to include the effects of dimensional tolerances and alignment, and in kinematics and joint mechanics predictions, it is desirable to include variability in patient geometry. As a result, many probabilistic studies begin with a deterministic or idealized geometry with varying material properties and loading input parameters. Recently, automated model generation and statistical shape modelling techniques have been successfully demonstrated in probabilistic applications.

Probabilistic analyses have traditionally evaluated a single performance measure. In many biomechanics applications, it is desirable to predict multiple performance measures (e.g. 6 degree-of-freedom kinematics, contact mechanics, etc.) and the measures are often a function of the motion cycle. These more complex analyses can be performed, but typically require custom interfacing between the probabilistic method and the model.

\subsection{Common tools}

To perform probabilistic analyses, there are dedicated probabilistic packages, e.g. Nessus (Southwest Research Institute, San Antonio, Texas), or probabilistic modules within other modelling packages, e.g. iSight in Abaqus (Simulia, Providence, Rhode Island), PDS in Ansys (Ansys, Inc., Canonsburg, Pennsylvania), PamOpt in Pamcrash (ESI Group, Paris, France), and Adams (MSC, Santa Ana, California). The dedicated probabilistic packages can be linked with any analysis and tend to offer a wider variety of analysis capabilities and control, but also require interfacing with the model (e.g. the finite element model). The algorithms in Nessus (Southwest Research Institute, San Antonio, Texas) have undergone verification and validation with the methods presented in peer-reviewed journal publications [4, 6-8]. Modelling packages with probabilistic modules commonly implement Monte Carlo and response surface methods; several have improved efficiency by approximating response surfaces from derivatives of parameters, including material properties and even dimensional variability, within the solution methodology [9]. It is important to note that an understanding of the underlying probabilistic methods is critical in understanding the performance predictions and their accuracy. If a Monte Carlo simulation was performed on a response surface, for example, it is possible to have millions of Monte Carlo simulations, but the underlying accuracy of the solution is based on the number of analyses used to develop the response surface itself.

\section{STRUCTURAL RELIABILITY}

Probabilistic methods have traditionally been applied in applications of structural reliability in turbine discs [10], automobile and aerospace components $[\mathbf{1 1}, 12]$, and offshore structures [13]. Structural reliability applications typically evaluate failure referring to the likelihood that stresses in a component will exceed the strength of the component's material. Even when a safety factor, indicating that stresses are below the material's strength, is present, when uncertainty is considered failure may occur a small percentage of the time.

Early probabilistic studies in biomechanics assessed the structural reliability of orthopaedic components [14, 15]. Browne et al. applied reliability theory to aid in fracture mechanics-based life prediction procedures for a tibial tray component represented as a cantilever beam subjected to constant amplitude loading [14]. Dar et al. demonstrated how the Taguchi design of experiments and probabilistic methods could complement each other to account for uncertainties when predicting stresses with finite element analysis in a study of a fixation plate represented as a cantilever beam [15]. The effects of geometric tolerances and material property variability on stress and fatigue life were evaluated with an automated computational tool to perform geometric modifications of a hip stem by Easley et al. [3].

The complexity of the analyses expands considerably when the implant is considered within its construct, including the surrounding bone and bone cement. Nicolella et al. developed a three-dimensional model of an implanted cemented hip stem as the subject of a probabilistic study where variability in material properties and loading was considered in order to predict a probability of failure due to three separate cement failure modes [16]. Mehrez et al. used an idealized cylindrical finite element model to represent an implanted cemented hip stem in order 
to assess the most likely mode of failure and to identify which parameters had the largest contribution, where geometry, material properties, and loading were considered to be random variables [17]. Excellent agreement in results was noted for FORM and Monte Carlo probabilistic methods. Similarly, Bah and Browne [18] used an idealized cemented hip model to look at the effect of geometrical uncertainty on the cement stresses in the implanted hip. A response surface was created using LHS, which was benchmarked using Monte Carlo simulation; it was found that bone and prosthesis geometry played a significant role and it was suggested that these parameters should be accounted for in future probabilistic analyses. Bah et al. have evaluated changes in implant alignment and the corresponding effect on structural integrity using a mesh morphing technique [19]. The approach can efficiently generate models with geometric perturbations that can prove useful in optimization and probabilistic studies. Chang et al. combined optimization techniques with sensitivity analysis to demonstrate a robust design of a hip stem within a bone and cement construct by minimizing the bone remodelling signal [20]. Latin hypercube and a statistically based meta-model, based on a design dimensional, patient-specific load, surgical placement, and environmental input parameter, were used to develop a robust acetabular cup design efficiently [21].

Recent studies have applied probabilistic approaches to account for fatigue and micromotion failure mechanisms with uncertainty in material behaviour, microstructural features, and damage accumulation. Jeffers et al. applied a Monte Carlobased approach to a finite element and continuum mechanics model to predict the locations of pores and evaluated fatigue performance of coupons and an implanted femoral construct [22, 23]. Pidaparti $e t$ al. developed a Monte Carlo-based damage accumulation model for microcracks related to bone fatigue, including a microdamage parameter with impact on fatigue life and bone stiffness loss [24]. A Monte Carlo analysis was performed on a hip stem construct to evaluate the likelihood of instability or micromotion based on uncertainty in the bone material properties, body weight loading, implant size, and the region of contact [25]. Considering uncertainty in bone property, loading, and also component alignment in a hip stem construct, Dopico-Gonzalez et al. applied probabilistic methods to find that the volume of strained bone was sensitive to the implant version angle, bone mod- ulus, and applied load; good agreement was demonstrated for Monte Carlo and Latin hypercube sampling [26, 27]. In a study evaluating the response of three femurs to two types of implants, one with a stem and one without a stem, Dopico-Gonzalez et al. showed that micromotion in both implants was most sensitive to implant positioning parameters (Fig. 3) [28]. The stemless design demonstrated much higher sensitivities, although the order of sensitivity changed with femur characteristics. Considering the amount of bonding at the stem-cement interface as a probabilistic parameter, Pérez et al. characterized the impact of interface bonding on the region, mechanisms, and likelihood of failure [29].

When considering bone structures, local material properties can be applied based on density measurements from computerized tomography (CT) scan data. Automated techniques have been developed to build geometries and assign material properties to patient specific bones from CT scan data [30-32]. Radcliffe and Taylor applied perturbations of component alignment [33] and cement mantle thickness [34] to assess bone strain in a hip stem construct and, by using a population of 16 subjects, the analysis considered patient variability. Laz et al. included uncertainty in the density-to-modulus and density-to-strength relationships used in assigning bone material properties and predicted large amounts of variability in stress and risk in a proximal femur [35]. Monte Carlo and Latin hypercube approaches were implemented by Taddei et al. to evaluate the impact of uncertainty in geometry, density, and material properties of the bone tissue on bone displacement stresses and strains under compression and torsion loading [36]. Using correlation coefficients, the study reported that scaling of the geometry had the greatest impact on the performance measures.

\section{KINEMATICS}

In experimental studies, probed anatomical landmarks are commonly used to define coordinate frames in which kinematic motions are reported. Uncertainty in the identification and location of the anatomical landmarks were quantified by Della Croce et al. at intra- and interexaminer levels and led to variability in predicted kinematics in motion analysis $[\mathbf{3 7}, \mathbf{3 8}]$. Using ANOVA on kinematic data for the shoulder, de Groot characterized the relative contributions of palpation errors, motoric noise (kinematic repeatability), and intersubject differences [39]. While this study was able to quantify 

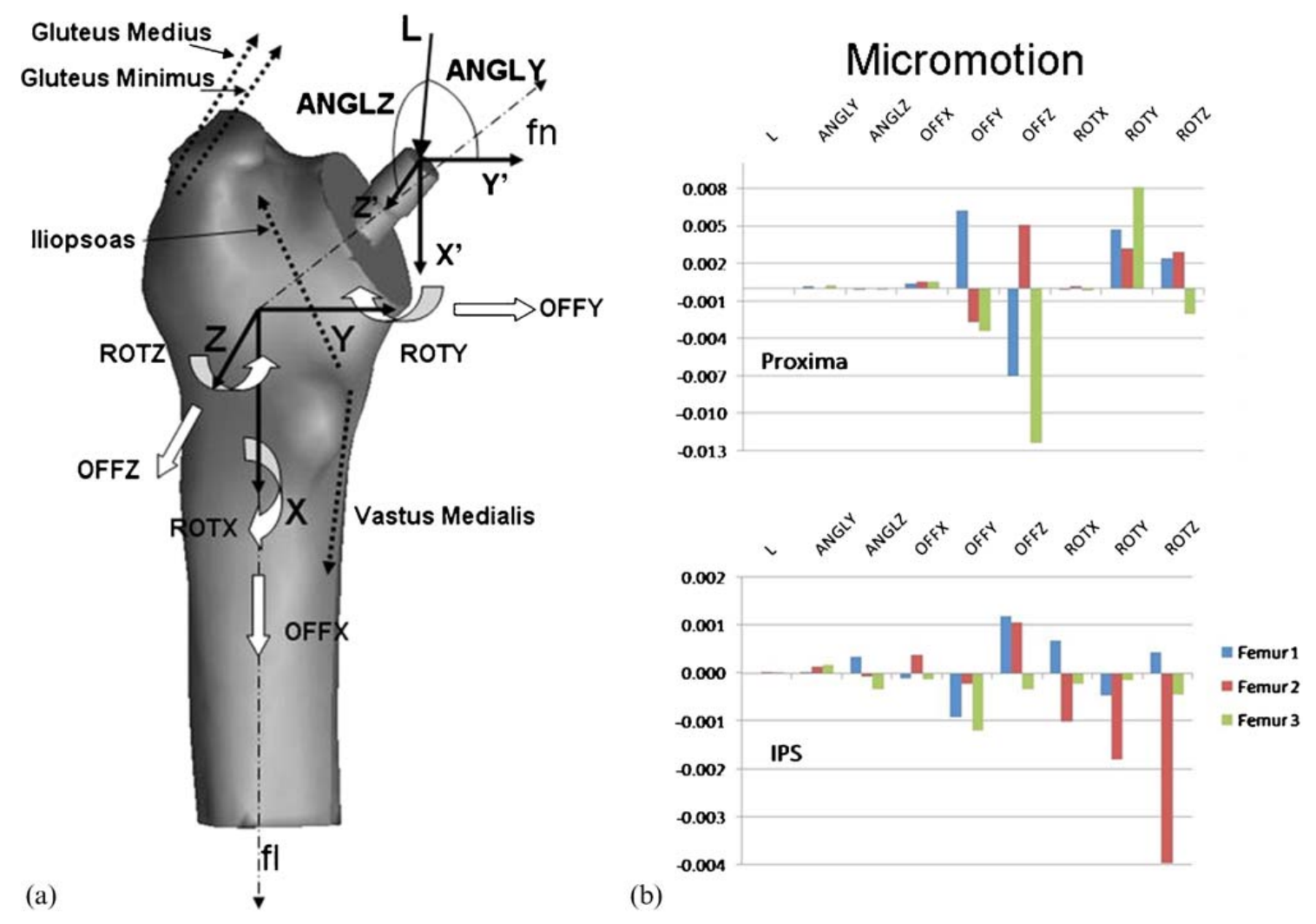

Fig. 3 Probabilistic application in a hip construct from Dopico-Gonzalez et al. [28]. Three bones were implanted with two uncemented designs: (a) variable parameters including implant orientation, muscle forces, and loading, (b) a sensitivity analysis showing the effect of variable parameters on micromotion for each bone and design

the impact of various uncertainties, it required knowledge of the results a priori.

Alternatively, probabilistic approaches have introduced uncertainty in the anatomical landmark locations and characterized the impact on reference frame and kinematic description $[40,41]$. Morton et $a l$. predicted the 1 and 99 per cent bounds for tibiofemoral and patellofemoral kinematics during gait and identified the most important landmarks contributing to the variability [41]. Similarly, in the shoulder, Langenderfer et al. predicted variability in reported shoulder Euler angles for 10 subjects due to anatomical landmark location uncertainty and also demonstrated that the important parameters were consistent between subjects [40].

\section{JOINT MECHANICS}

Kinematic data from motion studies, fluoroscopy, and experiments are often used to drive inverse dynamics models to predict intersegmental forces and moments or finite element analyses to predict joint contact mechanics. Sensitivity of joint kinetics during gait has been investigated using non-probabilistic and probabilistic approaches. By applying ANOVA, uncertainty in body segment parameters, based on using various estimation models, resulted in significant differences of up to 20 per cent in the flexion-extension moment at the hip [42]. In a motion capture and inverse dynamics study, Holden and Stanhope showed that changing the knee centre location by $\pm 10 \mathrm{~mm}$ in the anteroposterior (AP) direction did not greatly affect the shape of the knee moment patterns, but the moment magnitude was significantly impacted and even changed sign at the slower walking speeds [43].

More recently, Reinbolt et al. applied Monte Carlo analysis to characterize the uncertainty in inverse dynamics, including uncertainty in the joint parameters (axis positions and orientations) and inertial parameters (segment masses, mass centres, and moments of inertia) [44]. The study found that the predicted joint torques varied by up to 4 per cent of body weight $\times$ height and were impacted more by 2 joint parameters than inertial parameters. Langenderfer et al. reported similar findings in a small fraction of the number of analyses using efficient 
probabilistic techniques (AMV) and also presented sensitivity factors to identify the most important input parameters [45].

The sensitivity of joint mechanics predictions has been investigated with computational models by perturbing individual parameters, including defined axes, component alignment, and material representations for cartilage, ligaments, muscles, and intervertebral discs. By evaluating the transepicondylar and geometric centre axis, knee joint kinematics were shown to be highly sensitive to the selection of the flexion axis by Most et al. [46]. In a fluoroscopydriven finite element study, Fregly et al. characterized significant differences in contact force, pressure, and area based on small $\pm 0.1 \mathrm{~mm}$ or degree perturbations in the pose of total knee replacement (TKR) components [47].

Probabilistic analysis allows consideration of uncertainty in multiple parameters, including the potential impact of interaction effects. Under simulated gait conditions, uncertainty in component alignment (standard deviations of $0.5 \mathrm{~mm}$ and $0.5^{\circ}$ ), loading and experimental set-up was included

3 to predict 1-99 percentile envelopes of AP and IE kinematics and contact pressure $[48,49]$. The studies showed that efficient AMV results agreed closely with Monte Carlo results and demonstrated differences in the relative rank of the important input parameter for two TKR designs. Utilizing a similar probabilistic approach with Archard's law, Pal et al. presented an efficient platform for predicting implant wear and its variability [50]. Further, Strickland et al. demonstrated design-dependent correlations between passive laxity and active gait mechanics for a cruciate-retaining fixed-bearing TKR design [51]. Using Monte Carlo simulation on a rigid-body model, a good correlation was observed for kinematics and peak contact pressures with those of Laz et al. [49] under normal gait, and it was shown that a larger degree of output variability was possible through the incorporation of spring elements representing knee ligament restraint.

As soft tissue constraint naturally influences joint mechanics, numerous experimental and computational studies have attempted to characterize uncertainty in ligament material properties, reference strains, subject-to-subject differences in attachment site location, and overall passive joint laxity. Variability in ligament linear stiffness of more than 30 per cent of the mean has been reported from controlled experimental characterization tests [52-54]. Probabilistic representations of ligament stress-strain behaviour have been developed with a microstruc- tural model accounting for fibre recruitment to account for the 'toe' and linear regions [55] and with a collagen fibre model [56]. Ligament attachment sites are typically located by digitizing points in cadaver experiments or from imaging data. In experiments, the uncertainty is likely to be comparable to locating and digitizing anatomical landmarks, which resulted in standard deviations of up to $12.2 \mathrm{~mm}$ [38]. When using image data, there is additional uncertainty in defining attachment boundaries and differentiating between ligament and adjacent bony or other anatomical structures.

Experimentally measured force-displacement and torque-rotation knee laxity curves contain large amounts of variability [57-59]. For example, Markolf et al., in in vivo AP and IE laxity tests on 49 and 20 subjects, reported standard deviations of up to $2.7 \mathrm{~mm}$ and $12.1^{\circ}$ respectively [58]. Recognizing the potential impact of uncertainty, Weiss et al. [60] advocate performing sensitivity studies, especially when applying population averages to subjectspecific models. Recent studies have investigated the effects of uncertainty in the ligament mechanical properties on the predicted knee joint constraint (e.g. see references [61] to [63]). Other experimental [64-66] and computational [62] studies have shown that varying ligament attachment site locations by as little as $2 \mathrm{~mm}$ affected joint kinematics. Due to the expensive computational cost of exploring all combinations and levels of input parameter variations, constraint sensitivity predictions typically involved discrete changes in ligament input parameters (stiffness) and were focused on the cruciate ligaments under specific loading scenarios. Recently, Baldwin et al. [67] developed a probabilistic representation of knee ligament constraints, including uncertainty in ligament stiffness, reference strain, and attachment site (Fig. 4). The predicted laxity bounds showed that the efficient AMV method agreed closely with the Monte Carlo method, with a fourfold reduction in computation time and sensitivity factors identifying the critical properties agreed with reported ligament recruitment [67].

Probabilistic methods have also been applied in spine mechanics to assess the important factors affecting torque-rotation behaviour. Variability in annulus, nucleus, bone, and ligament material properties were included in a cervical spine model to predict the distribution of rotation due to an applied flexion-extension moment, sensitivity factors, and the risk of injury [69]. Ng and Teo studied the influence of material moduli uncertainty in cervical spine components on biomechanical re- 

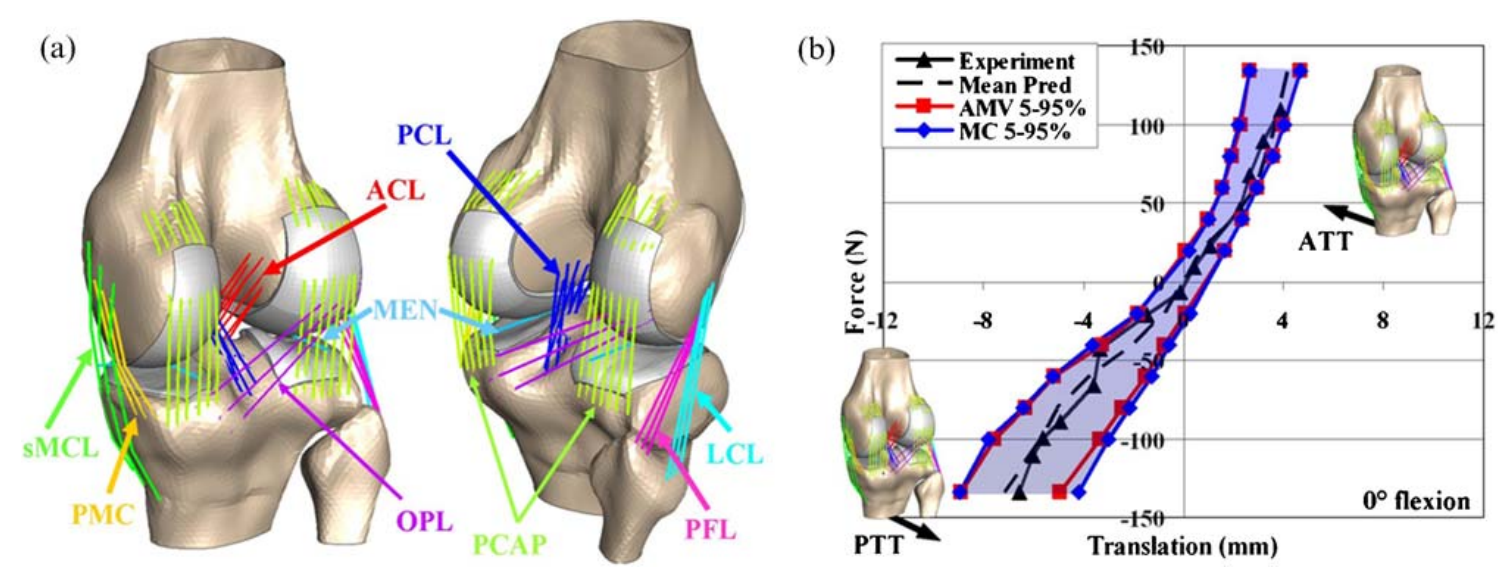

Fig. 4 Probabilistic prediction of knee laxity bounds including uncertainty in ligament stiffness, reference strain, and attachment site from Baldwin et al. [67]: (a) finite element model with ligament structures, (b) predicted laxity bounds (mean, 5 and 95 per cent) for anterioposterior translation at full extension for the Monte Carlo and AMV methods compared to experimental data $[\mathbf{6 8}]$

sponses and disc annulus stress using a threedimensional finite element model and Monte Carlo simulation methods [70]. Lee and Teo [71] used probabilistic sensitivity factors to identify the important bone, disc, and ligament material properties affecting sagittal rotation in the L2-L3 functional spinal unit (FSU). Highlighting computational efficiency both in the finite element formulation and in the probabilistic analysis, AMV-predicted torquerotation behaviour considering disc and ligament material variability in a natural lumbar spine FSU compared well with Monte Carlo results and required only 4 per cent of the analysis time [72]. Using Monte Carlo simulation and an L3-L5/S1 model, Rohlmann et al. evaluated the effect of disc replacement alignment, implant radius, facet spacing, and scar tissue on intervertebral rotation and facet loading [73].

\section{MUSCULOSKELETAL MODELLING}

In inverse-dynamics applications associated with musculoskeletal modelling, kinematics are applied to determine the muscle moment arms and forces generated for a specified motion. The uncertainty described in identifying anatomical landmarks and constructing coordinate frames and intersubject variability in kinematics will be carried forward to affect moment arm and muscle force predictions. In musculoskeletal modelling, moment arms are often evaluated and compared to experimental data as a verification step. Pal et al. have predicted substantial levels of variability in moment arms for muscles in the lower limb, considering uncertainty in the muscle attachment site, kinematic variability, and moment arm calculation technique [74].

In forward dynamic applications, muscle activation and a muscle model (e.g. Hill type) are used to predict muscle forces and ultimately kinematics. The sensitivity of forces predicted by a Hill-type muscle during a forward dynamic simulation were estimated by perturbing the individual muscle model inputs [75]. By varying each muscle parameter by \pm 50 per cent, Scovil and Ronsky found that muscle force was very sensitive to the parameters defining the length of the tendon (series elastic component), the force-length curve of the contractile element, and the maximum isometric force [75]. In a lowerlimb musculoskeletal model developed by McLean et al. [76], Monte Carlo perturbations on activation levels in the quadriceps and hamstrings and initial contact conditions were used to evaluate threedimensional loading at the knee. Compared to proposed injury mechanisms associated with sagittal plane forces, the predicted distribution of joint loading never exceeded the level expected for an ACL tear [77].

The upper extremity has been the subject of numerous probabilistic analyses due to the relative importance and uncertainty associated with muscle loading in the complex motions of the shoulder, elbow, and hand. Flieg et al. [77] performed a Monte Carlo simulation on a musculoskeletal model of the glenohumeral joint with uncertainty in muscle forces to compute the likelihood that the glenohumeral net reaction force would be directed to promote superior humeral head migration. Moving away 
from a single average musculoskeletal model, Langenderfer et al. accounted for subject variability with distributions characterizing musculoskeletal architecture and moment arm and showed that the probabilistic predictions of glenohumeral strength for healthy normal and subjects with rotator cuff tears explained the variability in measured values [78].

Probabilistic predictions with a biomechanical model of the elbow evaluated differences in joint force and torque distributions with and without long head biceps rupture [79]. To account for anatomic and kinematic variability in the hand, Valero-Cuevas and co-workers applied Monte Carlo simulation to forward and inverse musculoskeletal models of the thumb $[\mathbf{8 0}, \mathbf{8 1}]$. Valero-Cuevas et al. showed that robabilistic analysis incorporating variability and uncertainty in musculoskeletal parameters yielded differences between thumbtip force predictions and measurements, and non-physical results; accordingly, kinematic descriptions, not parameter uncertainty, were identified as the area requiring improvement $[\mathbf{8 0}]$. By converting anatomic variability into a standard robotics formulation, Santos and ValeroCuevas were able to identify a set of models accounting for anatomic and functional variability as an alternative to subject-specific models [81].

\section{REPRESENTATIONS OF SUBJECT GEOMETRY}

Understanding the potential impact of intersubject variability has been the focus of many prior works. Recent advances in imaging and geometry extraction techniques have facilitated the development of subject-specific models from CT and magnetic resonance (MR) scan data; however, the process remains largely manual and time consuming. As a result, many studies evaluated a small number of subject-specific models. Recently, intersubject variability has been considered by expanding the number of subject models. Based on reconstructed knee cartilage geometries from a dataset of MR images from 20 subjects, Connolly et al. characterized relationships between tibiofemoral cartilage thickness and a variety of anthopometric measures [82]. Using a population of 16 subjects, Radcliffe and Taylor considered patient variability $[\mathbf{3 3}, \mathbf{3 4}]$ and with co-workers provided an approach to determine the minimum sample size to evaluate a new implant design or to characterize statistically significant differences in design parameters [83].

An alternative approach for capturing intersubject variability is statistical shape modelling, which provides a representation of the variability present in a training set. Statistical or active shape models have used a point distribution model (PDM) to develop point-to-point correspondences between the instances in a training set [84-86]. Principal component analysis is often used to identify the common modes of variations, including the vectors along which the PDM changes [87]. As a result, the principal components can also be used to generate geometries of new 'subjects' using the statistical shape model. These techniques have been applied to develop shape models of individual bones [85] and statistical models that include both shape and bone density material property information (Fig. 5) [8891]. Utilizing an underlying template mesh and morphing mesh handles to structure locations in scan data for a new subject, Baldwin et al. demonstrated that mesh morphing can be applied efficiently to build a training set for statistical shape modelling [92]. Statistical shape modelling has been used to evaluate sizing and optimize coverage of implants on resected bone surfaces [93], to create subject-specific representations from incomplete or sparse datasets from less invasive methods (e.g. ultrasound) [94-96], and to investigate morphological correlation between multiple bones in a joint [97].

When used in conjunction with a probabilistic analysis technique, like Monte Carlo, statistical shape models can create a 'simulated' population of subjects that can be used for biomechanical evaluations, like fracture risk or the performance of an implant design. Bryan et al. applied a statistical model of shape and bone density (Fig. 5) based on a training set of 21 subjects in order to evaluate femoral neck fracture risk in a population of 1000 models $[\mathbf{9 1}$. The shape modelling approach provides an efficient means to evaluate larger numbers of subjects, including extremes, with the caveat that the variability in the population is based on the training set.

\section{SUMMARY}

This paper has demonstrated the wide range of applications of probabilistic modelling techniques within biomechanics. There is a long history of traditional computational and experimental investigations in the academic literature. These studies have undoubtedly been valuable in directing current practice in terms of implant design and performance assessment. At the same time, despite good lab practices, experimental data contain large amounts 


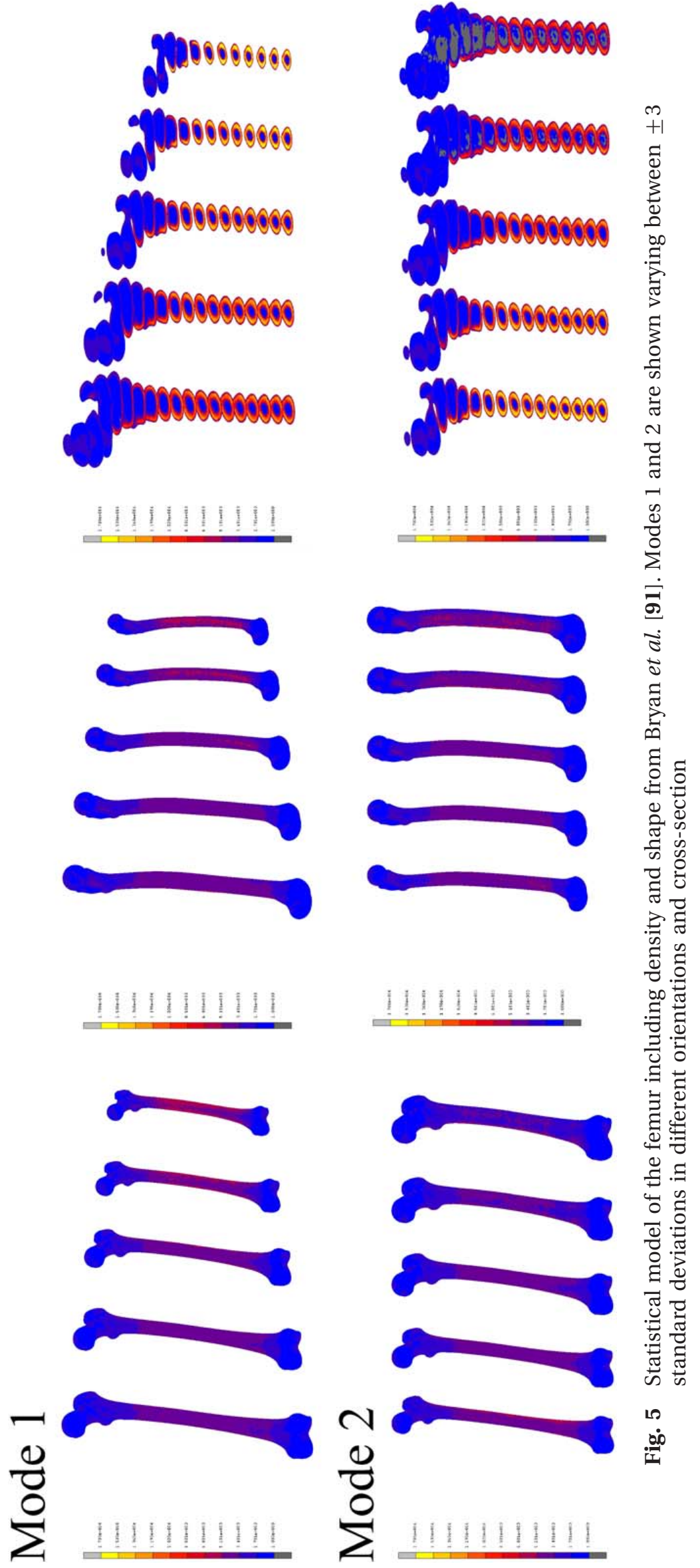


of variability due to inherent uncertainty. For example, even very similar tests on total knee replacements have resulted in a tenfold difference in wear rate, demonstrating that experimental procedures and sources of variability may not be fully controlled or understood [98, 99]. Many computational studies are focused on a small number of subjects and have not considered the high levels of variability inherent in the system under investigation. Given the rising demand for orthopaedic surgery and the amount of patient variability in factors such as age, size, and activity level, to name a few, there is a clear need for improved pre-clinical analysis methods that are computationally efficient and can provide additional useful information that has not been available to date using traditional deterministic methods.

A benefit of probabilistic analysis is the quantitative characterization of how an output measure is affected by variability in input parameters, including consideration of variable interaction effects and prediction of bounds of performance. In addition, a probabilistic analysis can provide valuable insight into the range of possible outcomes and the robustness of implant designs. It has also been shown that in many studies probabilistic techniques have been used to evaluate sensitivity of the output measure and to identify the most important parameters impacting performance. In alignment-related studies, knowledge of the critical parameters can influence surgical or experimental techniques.

With continuing improvements in computational resources and the desire for improved understanding of factors affecting clinical performance, it is expected that applications of probabilistic analysis will continue to grow in the biomechanics field. Applications of efficient probabilistic methods have been highlighted in numerous studies as providing accurate solutions in a small fraction of the computation time; however, benchmarking of these techniques against Monte Carlo simulation is recommended when applied to a new system.

While the benefits of probabilistic analysis may seem compelling, it should be noted that probabilistic approaches require many more trials, and therefore necessitate faster modelling methods than the deformable finite element models historically employed. Rigid-body modelling, even within the finite element platform, can provide this speed increase, usually with a minimal tradeoff in accuracy [100]. Increased computational power is one factor that has been highlighted as being crucial to the development of probabilistic analyses. However, it is the need for accurate, authentic data for input and output parameters that is arguably the most important factor in the development of probabilistic methods. Probabilistic analyses often make assumptions about an input distribution based on a limited amount of available data. It is important that conclusions based on predicted output distributions are valid and based on accurate and reliable input data. This underscores the importance of mutual understanding and close collaboration between the computational modeller and the experimentalist, so that potential sources of variability can be quantified during an experiment and accounted for in the analysis. Considering the various sources of uncertainty, probabilistic methods have the potential to aid in the design of implants that are robust to population variability, the development of relationships between component alignment and performance to assist clinicians, and more holistic assessments of joint mechanics, musculoskeletal loading, and pathologies.

(c) Authors 2010

\section{REFERENCES}

1 Haldar, A. and Mahadevan, S. Probability, reliability and statistical methods in engineering design, 2000 (John Wiley \& Sons, Inc., New York).

2 Melchers, R. E. Structural reliability analysis and prediction, 2001 (John Wiley \& Sons, Inc., New York).

3 Easley, S. K., Pal, S., Tomaszewski, P. R., Petrella, A. J., Rullkoetter, P. J., and Laz, P. J. Finite element-based probabilistic analysis tool for orthopaedic applications. Computer Methods and Programs in Biomedicine, 2007, 85(1), 32-40.

4 Wu, Y. T., Millwater, H. R., and Cruse, T. A. Advanced probabilistic structural-analysis method for implicit performance functions. Am. Inst. Aeronaut. Astronaut. J., 1990, 28(9), 1663-1669.

5 NESSUS Theoretical Manual, 2001 (Southwest Research Institute, San Antonio, Texas).

6 Riha, D. S., Millwater, H. R., and Thacker, B. H. Probabilistic structural analysis using a general purpose finite element program. Finite Elements in Analysis and Des., 1992, 11 (3), 201-211.

7 Riha, D. S., Thacker, B. H., Pleming, J. B., Walker, J. D., Mullin, S. A., Weiss, C. E., Rodriguez, E. A., and Leslie, P. O. Verification and validation for a penetration model using a deterministic and probabilistic design tool. Int. J. Impact Engng, 2006, 33(1-12), 681-690.

8 Thacker, B. H., Riha, D. S., Fitch, S. H. K., Huyse, L. J., and Pleming, J. B. Probabilistic engineering analysis using the NESSUS software. Struct. Safety, 2006, 28(1-2), 83-107. 
9 Reh, S., Beley, J.-D., Mukherjee, S., and Khor, E. H. Probabilistic finite element analysis using ANSYS. Struct. Safety, 2006, 28(1-2), 17-43.

10 Melis, M. E., Zaretsky, E. V., and August, R. Probabilistic analysis of aircraft gas turbine disk life and reliability. J. Propulsion and Power, 1999, 15(5), 658-666.

11 Kurth, R. E. and Woods, K. S. Probabilistic damage tolerant analyses for fatigue critical aircraft components, 1992, Anaheim, California, pp. 89-97.

12 Zhang, Y. and Liu, Q. Reliability-based design of automobile components. Proc. IMechE, Part D: J. Automobile Engineering, 2002, 216(D6), 455-471.

13 Langley, R. S. and McWilliam, S. Stochastic methods for offshore structures. In Chapter 6 in Mathematical models for structural reliability analysis (Eds F. Casciati and J. B. Roberts), 1996, ch. 6, pp. 287-341 (CRC Press, Boca Raton, Florida).

14 Browne, M., Langley, R. S., and Gregson, P. J. Reliability theory for load bearing biomedical implants. Biomaterials, 1999, 20(14), 1285-1292.

15 Dar, F. H., Meakin, J. R., and Aspden, R. M. Statistical methods in finite element analysis. J. Biomechanics, 2002, 35(9), 1155-1161.

16 Nicolella, D. P., Thacker, B. H., Katoozian, H., and Davy, D. T. The effect of three-dimensional shape optimization on the probabilistic response of a cemented femoral hip prosthesis. J. Biomechanics, 2006, 39(7), 1265-1278.

17 Mehrez, L., Bah, M. T., and Browne, M. Reliability analysis: a new approach to assess the performance of a cemented total hip replacement. In the 7th International Conference on Engineering Structural Integrity Assessment (ESIA7), 2004 Manchester, UK.

18 Bah, M. T. and Browne, M. Effect of geometrical uncertainty on cemented hip implant structural integrity. Trnas. ASME, J. Biomech. Engng, 2009, $131(5), 054501$.

19 Bah, M. T., Nair, P. B., and Browne, M. Mesh morphing for finite element analysis of implant positioning in cementless total hip replacements. Med. Engng Physics (in press) (DOI:10.1016/ j.medengphy.2009.08.001).

20 Chang, P. B., Williams, B. J., Bhalla, K. S. B., Belknap, T. W., Santner, T. J., Notz, W. I., and Bartel, D. L. Design and analysis of robust total joint replacements: finite element model experiments with environmental variables. Trans. ASME, J. Biomech. Engng, 2001, 123(3), 239-246.

21 Ong, K. L., Lehman, J., Notz, W. I., Santner, T. J., and Bartel, D. L. Acetabular cup geometry and bone-implant interference have more influence on initial periprosthetic joint space than joint loading and surgical cup insertion. Trans. ASME, J. Biomech. Engng, 2006, 128(2), 169-175.

22 Jeffers, J. R., Browne, M., Lennon, A. B., Prendergast, P. J., and Taylor, M. Cement mantle fatigue failure in total hip replacement: experi- mental and computational testing. J. Biomechanics, 2007, 40(7), 1525-1533.

23 Jeffers, J. R., Browne, M., Roques, A., and Taylor, M. On the importance of considering porosity when simulating the fatigue of bone cement. Trans. ASME, J. Biomech. Engng, 2005, 127(4), 563570.

24 Pidaparti, R. M., Wang, Q. Y., and Burr, D. B. Modeling fatigue damage evolution in bone. BioMed. Mater. Engng, 2001, 11(2), 69-78.

25 Viceconti, M., Brusi, G., Pancanti, A., and Cristofolini, L. Primary stability of an anatomical cementless hip stem: a statistical analysis. J. Biomechanics, 2006, 39(7), 1169-1179.

26 Dopico-Gonzalez, C., New, A. M., and Browne, M. Probabilistic analysis of an uncemented total hip replacement. Med. Engng Physics, 2009, 31(4), 470-476.

27 Dopico-Gonzalez, C., New, A. M., and Browne, M. A computational tool for the probabilistic finite element analysis of an uncemented total hip replacement considering variability in bone-implant version angle. Computer Methods in Biomechanics and Biomed. Engng (in press) (DOI: 10.1080/10255840902911536).

28 Dopico-Gonzalez, C., New, A. M., and Browne, M. Probabilistic finite element analysis of the uncemented hip replacement - effect of femur characteristics and implant geometry. J. Biomechanics (in press) (DOI:10.1016/j.jbiomech.2009. 09.039).

29 Pérez, M. A., Grasa, J., García-Aznar, J. M., Bea, J. A., and Doblaré, M. Probabilistic analysis of the influence of the bonding degree of the stemcement interface in the performance of cemented hip prostheses. J. Biomechanics, 2006, 39(10), 18591872.

30 Perillo-Marcone, A., Alonso-Vazquez, A., and Taylor, M. Assessment of the effect of mesh density on the material property discretisation within QCT based FE models: a practical example using the implanted proximal tibia. Computer Methods in Biomechanics and Biomed. Engng, 2003, 6(1), 17-26.

31 Taddei, F., Cristofolini, L., Martelli, S., Gill, H. S., and Viceconti, M. Subject-specific finite element models of long bones: an in vitro evaluation of the overall accuracy. J. Biomechanics, 2006, 39(13), 2457-2467.

32 Viceconti, M., Davinelli, M., Taddei, F., and Cappello, A. Automatic generation of accurate subject-specific bone finite element models to be used in clinical studies. J. Biomechanics, 2004, 37(10), 1597-1605.

33 Radcliffe, I. A. and Taylor, M. Investigation into the effect of varus-valgus orientation on load transfer in the resurfaced femoral head: a multifemur finite element analysis. Clin. Biomechanics, 2007, 22(7), 780-786.

34 Radcliffe, I. A. and Taylor, M. Investigation into the affect of cementing techniques on load 
transfer in the resurfaced femoral head: a multifemur finite element analysis. Clin. Biomechanics, 2007, 22(4), 422-430.

35 Laz, P. J., Stowe, J. Q., Baldwin, M. A., Petrella, A. J., and Rullkoetter, P. J. Incorporating uncertainty in mechanical properties for finite element-based evaluation of bone mechanics. J. Biomechanics, 2007, 40(13), 2831-2836.

36 Taddei, F., Martelli, S., Reggiani, B., Cristofolini, L., and Viceconti, M. Finite-element modeling of bones from CT data: sensitivity to geometry and material uncertainties. IEEE Trans. on Biomed. Engng, 2006, 53(11), 2194-2200.

37 Della Croce, U., Camomilla, V., Leardini, A., and Cappozzo, A. Femoral anatomical frame: assessment of various definitions. Med. Engng Physics, 2003, 25(5), 425-431.

38 Della Croce, U., Cappozzo, A., and Kerrigan, D. C. Pelvis and lower limb anatomical landmark calibration precision and its propagation to bone geometry and joint angles. Med. Biolog. Engng and Computing, 1999, 37(2), 155-161.

39 de Groot, J. H. The variability of shoulder motions recorded by means of palpation. Clin. Biomechanics, 1997, 12(7/8), 461-472.

40 Langenderfer, J. E., Rullkoetter, P. J., Mell, A. G., and Laz, P. J. A multi-subject evaluation of uncertainty in anatomical landmark location on shoulder kinematic description. Computer Methods in Biomechanics and Biomed. Engng, 2009, 12(2), 211-216.

41 Morton, N. A., Maletsky, L. P., Pal, S., and Laz, P. J. Effect of variability in anatomical landmark location on knee kinematic description. J. Orthop. Res., 2007, 25, 1221-1230.

42 Rao, G., Amarantini, D., Berton, E., and Favier, D. Influence of body segments' parameters estimation models on inverse dynamics solutions during gait. J. Biomechanics, 2006, 39(8), 15311536.

43 Holden, J. P. and Stanhope, S. J. The effect of variation in knee center location estimates on net knee joint moments. Gait and Posture, 1998, 7, $1-6$.

44 Reinbolt, J. A., Haftka, R. T., Chmielewski, T. L., and Fregly, B. J. Are patient-specific joint and inertial parameters necessary for accurate inverse dynamics analyses of gait? IEEE Trans. on Biomed. Engng, 2007, 54(5), 782-793.

45 Langenderfer, J. E., Laz, P. J., Petrella, A. J., and Rullkoetter, P. J. An efficient probabilistic methodology for incorporating uncertainty in body segment parameters and anatomical landmarks in joint loadings estimated from inverse dynamics. Trans. ASME, J. Biomech. Engng, 2008, 130(1), 014502-014507.

46 Most, E., Axe, J., Rubash, H., and Li, G. Sensitivity of the knee joint kinematics calculation to selection of flexion axes. J. Biomechanics, 2004, 37(11), 1743-1748.
47 Fregly, B. J., Banks, S. A., D’Lima, D. D., Clifford, W., and Colwell, J. Sensitivity of knee replacement contact calculations to kinematic measurement errors. J. Orthop. Res., 2008, 26(9), 1173-1179.

48 Laz, P. J., Pal, S., Fields, A., Petrella, A. J., and Rullkoetter, P. J. Effects of knee simulator loading and alignment variability on predicted implant mechanics: a probabilistic study. J. Orthop. Res., 2006, 24(12), 2212-2221.

49 Laz, P. J., Pal, S., Halloran, J. P., Petrella, A. J., and Rullkoetter, P. J. Probabilistic finite element prediction of knee wear simulator mechanics. $J$. Biomechanics, 2006, 39(12), 2303-2310.

50 Pal, S., Haider, H., Laz, P. J., Knight, L. A., and Rullkoetter, P. J. Probabilistic computational modeling of total knee replacement wear. Wear, 2008, 264(7-8), 701-707.

51 Strickland, M. A., Browne, M., and Taylor, M. Could passive knee laxity be related to active gait mechanics? An exploratory computational biomechanical study using probabilistic methods. Computer Methods in Biomechanics and Biomed. Engng, 2009.

52 Race, A. and Amis, A. A. The mechanical properties of the 2 bundles of the human posterior cruciate ligament. J. Biomechanics, 1994, 27(1), 13-24.

53 Robinson, J. R., Bull, A. M. J., and Amis, A. A. Structural properties of the medial collateral ligament complex of the human knee. J. Biomechanics, 2005, 38(5), 1067-1074.

54 Chandrashekar, N., Mansouri, H., Slauterbeck, J., and Hashemi, J. Sex-based differences in the tensile properties of the human anterior cruciate ligament. J. Biomechanics, 2006, 39(16), 29432950.

55 Hurschler, C., Provenzano, P. P., and Vanderby Jr, R. Application of a probabilistic microstructural model to determine reference length and toe-to-linear region transition in fibrous connective tissue. Trans. ASME, J. Biomech. Engng, 2003, 125(3), 415-422.

56 Bontempi, M. Probabilistic model of ligaments and tendons: quasistatic linear stretching. Phys. Rev. E., 2009, 79(3).

57 Gollehon, D. L., Torzilli, P. A., and Warren, R. F. The role of the posterolateral and cruciate ligaments in the stability of the human knee - a biomechanical study. J. Bone Jt Surg. - Am. Vol., 1987, 69A(2), 233-242.

58 Markolf, K. L., Kochan, A., and Amstutz, H. C. Measurement of knee stiffness and laxity in patients with documented absence of the anterior cruciate ligament. J. Bone Jt Surg. - Am. Vol., 1984, 66A(2), 242-253.

59 Robinson, J. R., Bull, A. M. J., Thomas, R. R. D., and Amis, A. A. The role of the medial collateral ligament and posteromedial capsule in controlling knee laxity. Am. J. Sports Medicine, 2006, 34(11), 1815-1823. 
60 Weiss, J. A., Gardiner, J. C., Ellis, B. J., Lujan, T. J., and Phatak, N. S. Three-dimensional finite element modeling of ligaments: technical aspects. Med. Engng Physics, 2005, 27(10), 845-861.

61 Peña, E., Calvo, B., Martínez, M. A., and Doblaré, M. A three-dimensional finite element analysis of the combined behavior of ligaments and menisci in the healthy human knee joint. J. Biomechanics, 2006, 39(9), 1686-1701.

62 Beillas, P., Lee, S. W., Tashman, S., and Yang, K. H. Sensitivity of the tibio-femoral response to finite element modeling parameters. Computer Methods in Biomechanics and Biomed. Engng, 2007, 10(3), 209-221.

63 Mesfar, W. and Shirazi-Adl, A. Biomechanics of changes in ACL and PCL material properties or prestrains in flexion under muscle force - implications in ligament reconstruction. Computer Methods in Biomechanics and Biomed. Engng, 2006, 9(4), 201-209.

64 Bylski-Austrow, D. I., Grood, E. S., Hefzy, M. S., Holden, J. P., and Butler, D. L. Anterior cruciate ligament replacements: a mechanical study of femoral attachment location, flexion angle at tensioning, and initial tension. J. Orthop. Res., 1990, 8(4), 522-531.

65 Grood, E. S., Hefzy, M. S., and Lindenfield, T. N. Factors affecting the region of most isometric femoral attachments. 1. The posterior cruciate ligament. Am. J. Sports Medicine, 1989, 17(2), 197-207.

66 Hefzy, M. S., Grood, E. S., and Noyes, F. R. Factors affecting the region of most isometric femoral attachments. 2. The anterior cruciate ligament. Am. J. Sports Medicine, 1989, 17(2), 208-216.

67 Baldwin, M. A., Laz, P. J., Stowe, J. Q., and Rullkoetter, P. J. Efficient probabilistic representation of tibiofemoral soft tissue constraint. Computer Methods in Biomechanics and Biomed. Engng, 2009 (DOI: 10.1080/10255840902822550).

68 Li, G., Suggs, J., and Gill, T. The effect of anterior cruciate ligament injury on knee joint function under a simulated muscle load: a three-dimensional computational simulation. Ann. Biomed. Engng, 2002, 30(5), 713-720.

69 Thacker, B. H., Nicolella, D. P., Kumaresan, S., Yoganandan, N., and Pintar, F. A. Probabilistic finite element analysis of the cervical spine. In Mathematical Modeling and Scientific Computing, Turkey, 2001, pp. 12-21.

70 Ng, H. W. and Teo, E. C. Probabilistic design analysis of the influence of material property on the human cervical spine. J. Spinal Disorders and Techniques, 2004, 17(2), 123-133.

71 Lee, K. K. and Teo, E. C. Material sensitivity study on lumbar motion segment (L2-L3) under sagittal plane loadings using probabilistic method. J. Spinal Disorders and Techniques, 2005, 18(2), 163170.
72 Rao, M., Petrella, A. J., Baldwin, M. A., Laz, P. J., and Rullkoetter, P. J. Efficient probabilistic finite element modeling for evaluation of spinal mechanics. In Transactions of the 34th Annual Meeting of the Orthopaedic Research Society, Las Vegas, Nevada, 2009, p. 1771.

73 Rohlmann, A., Mann, A., Zander, T., and Bergmann, G. Effect of an artificial disc on lumbar spine biomechanics: a probabilistic finite element study. Eur. Spine J., 2009, 18(1), 89-97.

74 Pal, S., Langenderfer, J. E., Stowe, J. Q., Laz, P. J., Petrella, A. J., and Rullkoetter, P. J. Probabilistic modeling of knee muscle moment arms: effects of methods, origin-insertion, and kinematic variability. Ann. Biomed. Engng, 2007, 35(9), 1632-1642.

75 Scovil, C. Y. and Ronsky, J. L. Sensitivity of a Hillbased muscle model to perturbations in model parameters. J. Biomechanics, 2005.

76 McLean, S. G., Huang, X., Su, A., and van den Bogert, A. J. Sagittal plane biomechanics cannot injure the ACL during sidestep cutting. Clin. Biomechanics, 2004, 19(8), 828-838.

77 Flieg, N. G., Gatti, C. J., Doro, L. C., Langenderfer, J. E., Carpenter, J. E., and Hughes, R. E. A stochastic analysis of glenoid inclination angle and superior migration of the humeral head. Clin. Biomechanics, 2008, 23(5), 554-561.

78 Langenderfer, J., Carpenter, J., Johnson, M., An, K.-n., and Hughes, R. A probabilistic model of glenohumeral external rotation strength for healthy normals and rotator cuff tear cases. Ann. Biomed. Engng, 2006, 34(3), 465-476.

79 Langenderfer, J. E., Hughes, R. E., and Carpenter, J. E. A stochastic model of elbow flexion strength for subjects with and without long head biceps tear. Computer Methods in Biomechanics and Biomed. Engng, 2005, 8(5), 315-322.

80 Valero-Cuevas, F. J., Johanson, M. E., and Towles, J. D. Towards a realistic biomechanical model of the thumb: the choice of kinematic description may be more critical than the solution method or the variability/uncertainty in musculoskeletal parameters. J. Biomechanics, 2003, 36, 1019-1030.

81 Santos, V. J. and Valero-Cuevas, F. J. Reported anatomical variability naturally leads to multimodal distributions of Denavit-Hartenberg parameters for the human thumb. IEEE Trans. on Biomed. Engng, 2006, 53(2), 155-163.

82 Connolly, A., FitzPatrick, D., Moulton, J., Lee, J., and Lerner, A. Tibiofemoral cartilage thickness distribution and its correlation with anthropometric variables. Proc. IMechE, Part H: J. Engineering in Medicine, 2008, 222(H1), 29-39.

83 Radcliffe, I. A., Prescott, P., Man, H. S., and Taylor, M. Determination of suitable sample sizes for multi-patient based finite element studies. Med. Engng Physics, 2007, 29(10), 1065-1072.

84 Cootes, T. F., Taylor, C. J., Cooper, D. H., and Graham, J. Active shape models - their training 
and application. Computer Vision and Image Understanding, 1995, 61(1), 38-59.

85 Meller, S. and Kalender, W. A. Building a statistical shape model of the pelvis. Int. Congr. Ser., 2004, 1268, 561-566.

86 Behiels, G., Maes, F., Vandermeulen, D., and Suetens, P. Evaluation of image features and search strategies for segmentation of bone structures in radiographs using active shape models. Med. Image Analysis, 2002, 6(1), 47-62.

87 Jolliffe, I. T. Principal component analysis, edition 2, Springer Series in Statistics, 2002 (SpringerVerlag, New York).

88 Bredbenner, T. L. and Nicolella, D. P. Statistical shape and density based finite element modeling of the human proximal femur. In Transactions of the 33rd Annual Meeting of the Orthopaedic Research Society, San Francisco, California, 2008, p. 0305.

89 Bryan, R., Mohan, S., Hopkins, A., Nair, P., and Taylor, M. Generation of a statistical model of the whole femur incorporating shape and material property distribution. In Transactions of the 33rd Annual Meeting of the Orthopaedic Research Society, San Francisco, California, 2008, p. 0912.

90 Querol, L. B., Buchler, P., Rueckert, D., Nolte, L. P., and Ballester, M. A. G. Statistical finite element model for bone shape and biomechanical properties. Med. Image Computing and ComputerAssisted Intervention - MICCAI 2006, Pt 1, 2006, 4190, 405-411.

91 Bryan, R., Nair, P. B., and Taylor, M. Use of a statistical model of the whole femur in a large scale, multi-model study of femoral neck fracture risk. J. Biomechanics, 2009, 42, 2171-2176.

92 Baldwin, M. A., Langenderfer, J. E., Rullkoetter, P. J., and Laz, P. J. Development of subjectspecific and statistical shape models of the knee using an efficient segmentation and mesh-morphing approach. Computer Methods and Programs in Biomedicine (in press) (DOI:10.1016/j.cmpb.2009. 07.005).
93 Fitzpatrick, C., FitzPatrick, D., Lee, J., and Auger, D. Statistical design of unicompartmental tibial implants and comparison with current devices. Knee, 2007, 14(2), 138-144.

94 Barratt, D. C., Chan, C. S. K., Edwards, P. J., Penney, G. P., Slomczykowski, M., Carter, T. J., and Hawkes, D. J. Instantiation and registration of statistical shape models of the femur and pelvis using 3D ultrasound imaging. Med. Image Analysis, 2008, 12(3), 358-374.

95 Shim, V. B., Pitto, R. P., Streicher, R. M., Hunter, P. J., and Anderson, I. A. Development and validation of patient-specific finite element models of the hemipelvis generated from a sparse CT data set. Trans ASME, J. Biomech. Engng, 2008, $130(5)$.

96 Rajamani, K. T., Styner, M. A., Talib, H., Zheng, G., Nolte, L. P., and Gonzalez Ballester, M. A. Statistical deformable bone models for robust 3D surface extrapolation from sparse data. Med. Image Analysis, 2007, 11(2), 99-109.

97 Yang, Y. M., Rueckert, D., and Bull, A. M. J. Predicting the shapes of bones at a joint: application to the shoulder. Computer Methods in Biomechanics and Biomed. Engng, 2008, 11(1), 19-30.

98 Essner, A., Herrera, L., Yau, S.-S., Wang, A., Dumbleton, J. H., and Manley, M. T. Sequentially crosslinked and annealed UHMWPE knee wear debris. In Transactions of the 51st Annual Meeting of the Orthopaedic Research Society, Washington, DC, 2005, p. 0071.

99 Williams, P. A., Tsukamoto, R., and Clarke, I. C. Wear debris from sequentially crosslinked and crosslinked PE in a knee simulator model. In Transactions of the 54th Annual Meeting of the Orthopaedic Research Society, San Francisco, California, 2008, p. 0218.

100 Halloran, J. P., Easley, S. K., Petrella, A. J., and Rullkoetter, P. J. Comparison of deformable and elastic foundation finite element simulations for predicting knee replacement mechanics. Trans. ASME, J. Biomech. Engng, 2005, 127(5), 813-818. 